\title{
On new inequalities of Hermite- Hadamard-Fejer type for harmonically convex functions via fractional integrals
}

\author{
Mehmet Kunt ${ }^{\text {* }}$, Imdat Işcan ${ }^{2}$, Nazlı Yazıcı ${ }^{1}$ and Uğur Gözütok ${ }^{1}$
}

*Correspondence:
mkunt@ktu.edu.tr
${ }^{1}$ Department
of Mathematics, Faculty
of Sciences, Karadeniz
Technical University, Trabzon,
Turkey
Full list of author information
is available at the end of the
article

*Correspondence: mkunt@ktu.edu.tr

of Mathematics, Faculty

of Sciences, Karadeniz

Turkey

\begin{abstract}
In this paper, firstly, new Hermite-Hadamard type inequalities for harmonically convex functions in fractional integral forms are given. Secondly, Hermite-Hadamard-Fejer inequalities for harmonically convex functions in fractional integral forms are built. Finally, an integral identity and some Hermite-Hadamard-Fejer type integral inequalities for harmonically convex functions in fractional integral forms are obtained. Some results presented here provide extensions of others given in earlier works.
\end{abstract}

Keywords: Hermite-Hadamard inequality, Hermite-Hadamard-Fejer inequality, Riemann-Liouville fractional integrals, Harmonically convex functions

Mathematics Subject Classification: 26A51, 26A33, 26D10

\section{Background}

Let $f: I \subset \mathbb{R} \rightarrow \mathbb{R}$ be a convex function defined on the interval $I$ of real numbers and $a, b \in I$. The inequality

$$
f\left(\frac{a+b}{2}\right) \leq \frac{1}{b-a} \int_{a}^{b} f(x) d x \leq \frac{f(a)+f(b)}{2}
$$

is well known in the literature as Hermite-Hadamard's inequality (Hadamard 1893; Hermite 1883).

The most well-known inequalities related to the integral mean of a convex function $f$ are the Hermite Hadamard inequalities or their weighted versions, the so-called Hermite-Hadamard-Fejér inequalities.

Fejér (1906) established the following Fejér inequality which is the weighted generalization of Hermite-Hadamard inequality (1):

Theorem 1 Let $f:[a, b] \rightarrow \mathbb{R}$ be a convex function. Then the inequality

$$
f\left(\frac{a+b}{2}\right) \int_{a}^{b} g(x) d x \leq \int_{a}^{b} f(x) g(x) d x \leq \frac{f(a)+f(b)}{2} \int_{a}^{b} g(x) d x
$$

holds, where $g:[a, b] \rightarrow \mathbb{R}$ is nonnegative, integrable and symmetric to $(a+b) / 2$.

(c) 2016 The Author(s). This article is distributed under the terms of the Creative Commons Attribution 4.0 International License (http://creativecommons.org/licenses/by/4.0/), which permits unrestricted use, distribution, and reproduction in any medium, provided you give appropriate credit to the original author(s) and the source, provide a link to the Creative Commons license, and indicate if changes were made. 
For some results which generalize, improve and extend the inequalities (1) and (2) see Bombardelli and Varošanec (1869), İşcan (2013a, 2014c), Minculete and Mitroi (2012), Sarıkaya (2012), Tseng et al. (2011).

We recall the following inequality and special functions which are known as Beta and hypergeometric function respectively:

$$
\begin{aligned}
\beta(x, y) & =\frac{\Gamma(x) \Gamma(y)}{\Gamma(x+y)}=\int_{0}^{1} t^{x-1}(1-t)^{y-1} d t, \quad x, y>0, \\
{ }_{2} F_{1}(a, b ; c ; z) & =\frac{1}{\beta(b, c-b)} \int_{0}^{1} t^{b-1}(1-t)^{c-b-1}(1-z t)^{-a} d t, \\
c & >b>0,|z|<1 \text { (see Kilbas et al. 2006). }
\end{aligned}
$$

Lemma 1 (Prudnikov et al. 1981; Wang et al. 2013) For $0<\alpha \leq 1$ and $0 \leq a<b$ we have

$$
\left|a^{\alpha}-b^{\alpha}\right| \leq(b-a)^{\alpha} .
$$

The following definitions and mathematical preliminaries of fractional calculus theory are used further in this paper.

Definition 1 (Kilbas et al. 2006) Let $f \in L[a, b]$. The Riemann-Liouville integrals $J_{a+}^{\alpha} f$ and $J_{b-}^{\alpha} f$ of oder $\alpha>0$ with $a \geq 0$ are defined by

$$
J_{a+}^{\alpha} f(x)=\frac{1}{\Gamma(\alpha)} \int_{a}^{x}(x-t)^{\alpha-1} f(t) d t, \quad x>a
$$

and

$$
J_{b-}^{\alpha} f(x)=\frac{1}{\Gamma(\alpha)} \int_{x}^{b}(t-x)^{\alpha-1} f(t) d t, \quad x<b
$$

respectively, where $\Gamma(\alpha)$ is the Gamma function defined by $\Gamma(\alpha)=\int_{0}^{\infty} e^{-t} t^{\alpha-1} d t$ and $J_{a+}^{0} f(x)=J_{b-}^{0} f(x)=f(x)$.

Because of the wide application of Hermite-Hadamard type inequalities and fractional integrals, many researchers extend their studies to Hermite-Hadamard type inequalities involving fractional integrals not limited to integer integrals. Recently, more and more Hermite-Hadamard inequalities involving fractional integrals have been obtained for different classes of functions; see Dahmani (2010), İșcan (2013b, 2014a), İșcan and Wu (2014), Mihai and Ion (2014), Sarıkaya et al. (2013), Wang et al. (2012), Wang et al. (2013). İșcan (2014b) can defined the so-called harmonically convex functions and established following Hermite-Hadamard type inequality for them as follows:

Definition 2 Let $I \subset \mathbb{R} \backslash\{0\}$ be a real interval. A function $f: I \rightarrow \mathbb{R}$ is said to be harmonically convex, if

$$
f\left(\frac{x y}{t x+(1-t) y}\right) \leq t f(y)+(1-t) f(x)
$$


for all $x, y \in I$ and $t \in[0,1]$. If the inequality in (3) is reversed, then $f$ is said to be harmonically concave.

Theorem 2 (İșcan 2014b) Let $f: I \subset \mathbb{R} \backslash\{0\} \rightarrow \mathbb{R}$ be a harmonically convex function and $a, b \in$ I. If $f \in L[a, b]$ then the following inequalities holds:

$$
f\left(\frac{2 a b}{a+b}\right) \leq \frac{a b}{b-a} \int_{a}^{b} \frac{f(x)}{x^{2}} d x \leq \frac{f(a)+f(b)}{2} .
$$

Latif et al. (2015) gave the following definition:

Definition 3 A function $g:[a, b] \subseteq \mathbb{R} \backslash\{0\} \rightarrow \mathbb{R}$ is said to be harmonically symmetric with respect to $2 a b / a+b$ if

$$
g(x)=g\left(\frac{1}{\frac{1}{a}+\frac{1}{b}-\frac{1}{x}}\right)
$$

holds for all $x \in[a, b]$.

Chen and Wu (2014) presented a Hermite-Hadamard-Fejer type inequality for harmonically convex functions as follows:

Theorem 3 Let $f: I \subseteq \mathbb{R} \backslash\{0\} \rightarrow \mathbb{R}$ be a harmonically convex function and $a, b \in I$. If $f \in L[a, b]$ and $g:[a, b] \subseteq \mathbb{R} \backslash\{0\} \rightarrow \mathbb{R}$ is nonnegative, integrable and harmonically symmetric with respect to $\frac{2 a b}{a+b}$, then

$$
\begin{aligned}
f\left(\frac{2 a b}{a+b}\right) \int_{a}^{b} \frac{g(x)}{x^{2}} d x & \leq \int_{a}^{b} \frac{f(x) g(x)}{x^{2}} d x \\
& \leq \frac{f(a)+f(b)}{2} \int_{a}^{b} \frac{g(x)}{x^{2}} d x .
\end{aligned}
$$

In this paper, we give new Hermite-Hadamard type inequalities for harmonically convex functions in fractional integral forms. We establish new Hermite-Hadamard-Fejer inequalities for harmonically convex functions in fractional integral forms. We obtain an integral identity and some Hermite-Hadamard-Fejer type integral inequalities for harmonically convex functions in fractional integral forms.

\section{Main results}

Throughout this section, we write $\|g\|_{\infty}=\sup _{t \in[a, b]}|g(t)|$, for the continuous function $g:[a, b] \rightarrow \mathbb{R}$.

Lemma 2 If $g:[a, b] \subseteq \mathbb{R} \backslash\{0\} \rightarrow \mathbb{R}$ is integrable and harmonically symmetric with respect to $\frac{2 a b}{a+b}$, then

$$
J_{\frac{a+b}{2 a b}+}^{\alpha}(g \circ h)(1 / a)=J_{\frac{a+b}{2 a b}-}^{\alpha}(g \circ h)(1 / b)=\frac{1}{2}\left[\begin{array}{c}
J_{\frac{a+b}{2 a b}+}^{\alpha}(g \circ h)(1 / a) \\
+J_{\frac{a+b}{2 a b}-}^{\alpha}(g \circ h)(1 / b)
\end{array}\right]
$$


with $\alpha>0$ and $h(x)=1 / x, x \in\left[\frac{1}{b}, \frac{1}{a}\right]$.

Proof Since $g$ is harmonically symmetric with respect to $\frac{2 a b}{a+b}$, using Definition 3 we have $g\left(\frac{1}{x}\right)=g\left(\frac{1}{\left(\frac{1}{a}\right)+\left(\frac{1}{b}\right)-x}\right)$, for all $x \in\left[\frac{1}{b}, \frac{1}{a}\right]$. Hence, in the following integral setting $t=\left(\frac{1}{a}\right)+\left(\frac{1}{b}\right)-x$ and $d t=-d x$ gives

$$
\begin{aligned}
J_{\frac{a+b}{2 a b}+}^{\alpha}(g \circ h)(1 / a) & =\frac{1}{\Gamma(\alpha)} \int_{\frac{a+b}{2 a b}}^{\frac{1}{a}}\left(\frac{1}{a}-t\right)^{\alpha-1} g\left(\frac{1}{t}\right) d t \\
& =\frac{1}{\Gamma(\alpha)} \int_{\frac{1}{b}}^{\frac{a+b}{2 a b}}\left(x-\frac{1}{b}\right)^{\alpha-1} g\left(\frac{1}{(1 / a)+(1 / b)-x}\right) d x \\
& =\frac{1}{\Gamma(\alpha)} \int_{\frac{1}{b}}^{\frac{a+b}{2 a b}}\left(x-\frac{1}{b}\right)^{\alpha-1} g\left(\frac{1}{x}\right) d x=J_{\frac{a+b}{2 a b}-}^{\alpha}(g \circ h)(1 / b) .
\end{aligned}
$$

This completes the proof.

Theorem 4 Let $f: I \subseteq(0, \infty) \rightarrow \mathbb{R}$ be a function such that $f \in L[a, b]$, where $a, b \in I$. If $f$ is a harmonically convex function on $[a, b]$, then the following inequalities for fractional integrals holds:

$$
\begin{aligned}
f\left(\frac{2 a b}{a+b}\right) & \leq \frac{\Gamma(\alpha+1)}{2^{1-\alpha}}\left(\frac{a b}{b-a}\right)^{\alpha}\left\{\begin{array}{c}
J_{\frac{a+b}{2 a b}+}^{\alpha}(f \circ h)(1 / a) \\
+J_{\frac{a+b}{2 a b}-}^{\alpha}(f \circ h)(1 / b)
\end{array}\right\} \\
& \leq \frac{f(a)+f(b)}{2}
\end{aligned}
$$

with $\alpha>0$ and $h(x)=1 / x, x \in\left[\frac{1}{b}, \frac{1}{a}\right]$.

Proof Since $f$ is a harmonically convex function on $[a, b]$, we have for all $t \in[0,1]$

$$
\begin{aligned}
f\left(\frac{2 a b}{a+b}\right) & =f\left(\frac{2\left(\frac{a b}{t a+(1-t) b}\right)\left(\frac{a b}{t b+(1-t) a}\right)}{\left(\frac{a b}{t a+(1-t) b}\right)+\left(\frac{a b}{t b+(1-t) a}\right)}\right) \\
& \leq \frac{f\left(\frac{a b}{t a+(1-t) b}\right)+f\left(\frac{a b}{t b+(1-t) a}\right)}{2} .
\end{aligned}
$$

Multiplying both sides of (7) by $2 t^{\alpha-1}$, then integrating the resulting inequality with respect to $t$ over $\left[0, \frac{1}{2}\right]$, we obtain

$$
\begin{aligned}
2 f & \left(\frac{2 a b}{a+b}\right) \int_{0}^{\frac{1}{2}} t^{\alpha-1} d t \\
& \leq \int_{0}^{\frac{1}{2}} t^{\alpha-1}\left[f\left(\frac{a b}{t a+(1-t) b}\right)+f\left(\frac{a b}{t b+(1-t) a}\right)\right] d t \\
& =\int_{0}^{\frac{1}{2}} t^{\alpha-1} f\left(\frac{a b}{t a+(1-t) b}\right) d t+\int_{0}^{\frac{1}{2}} t^{\alpha-1} f\left(\frac{a b}{t b+(1-t) a}\right) d t .
\end{aligned}
$$


Setting $x=\frac{t b+(1-t) a}{a b}$ and $d x=\left(\frac{b-a}{a b}\right) d t$ gives

$$
\begin{aligned}
\frac{2^{1-\alpha}}{\alpha} f\left(\frac{2 a b}{a+b}\right) & \leq\left(\frac{a b}{b-a}\right)^{\alpha}\left\{\begin{array}{c}
\int_{\frac{1}{b}}^{\frac{a+b}{2 a b}}\left(x-\frac{1}{b}\right)^{\alpha-1} f\left(\frac{1}{\frac{1}{a}+\frac{1}{b}-x}\right) d x \\
+\int_{\frac{1}{b}}^{\frac{a+b}{2 a b}}\left(x-\frac{1}{b}\right)^{\alpha-1} f\left(\frac{1}{x}\right) d x
\end{array}\right\} \\
& =\left(\frac{a b}{b-a}\right)^{\alpha}\left\{\begin{array}{c}
\int_{\frac{a+b}{2 a b}}^{\frac{1}{a}}\left(\frac{1}{a}-x\right)^{\alpha-1} f\left(\frac{1}{x}\right) d x \\
+\int_{\frac{1}{b}}^{\frac{a+b}{2 a b}}\left(x-\frac{1}{b}\right)^{\alpha-1} f\left(\frac{1}{x}\right) d x
\end{array}\right\} \\
& =\left(\frac{a b}{b-a}\right)^{\alpha} \Gamma(\alpha)\left[J_{\frac{a+b}{2 a b}+}^{\alpha}(f \circ h)(1 / a)+J_{\frac{a+b}{2 a b}-}^{\alpha}(f \circ h)(1 / b)\right]
\end{aligned}
$$

and the first inequality is proved.

For the proof of the second inequality in (6), we first note that, if $f$ is a harmonically convex function, then, for all $t \in[0,1]$, it yields

$$
f\left(\frac{a b}{t a+(1-t) b}\right)+f\left(\frac{a b}{t b+(1-t) a}\right) \leq f(a)+f(b) .
$$

Then multiplying both sides of (8) by $t^{\alpha-1}$ and integrating the resulting inequality with respect to $t$ over $\left[0, \frac{1}{2}\right]$, we obtain

$$
\begin{aligned}
& \int_{0}^{\frac{1}{2}} t^{\alpha-1} f\left(\frac{a b}{t a+(1-t) b}\right) d t+\int_{0}^{\frac{1}{2}} t^{\alpha-1} f\left(\frac{a b}{t b+(1-t) a}\right) d t \\
& \quad \leq[f(a)+f(b)] \int_{0}^{\frac{1}{2}} t^{\alpha-1} d t=\frac{2^{1-\alpha}}{\alpha} \frac{f(a)+f(b)}{2}
\end{aligned}
$$

i.e.

$$
\begin{aligned}
& \left(\frac{a b}{b-a}\right)^{\alpha} \Gamma(\alpha)\left[J_{\frac{a+b}{2 a b}+}^{\alpha}(f \circ h)(1 / a)+J_{\frac{a+b}{2 a b}-}^{\alpha}(f \circ h)(1 / b)\right] \\
& \quad \leq \frac{2^{1-\alpha}}{\alpha}\left(\frac{f(a)+f(b)}{2}\right) .
\end{aligned}
$$

The proof is completed.

Theorem 5 Let $f:[a, b] \rightarrow \mathbb{R}$ be a harmonically convex function with $a<b$ and $f \in L[a, b]$. If $g:[a, b] \rightarrow \mathbb{R}$ is nonnegative, integrable and harmonically symmetric with respect to $\frac{2 a b}{a+b}$, then the following inequalities for fractional integrals holds:

$$
\begin{aligned}
& f\left(\frac{2 a b}{a+b}\right)\left[J_{\frac{a+b}{2 a b}+}^{\alpha}(g \circ h)(1 / a)+J_{\frac{a+b}{2 a b}-}^{\alpha}(g \circ h)(1 / b)\right] \\
& \quad \leq\left[J_{\frac{a+b}{2 a b}+}^{\alpha}(f g \circ h)(1 / a)+J_{\frac{a+b}{2 a b}-}^{\alpha}(f g \circ h)(1 / b)\right] \\
& \quad \leq \frac{f(a)+f(b)}{2}\left[J_{\frac{a+b}{2 a b}+}^{\alpha}(g \circ h)(1 / a)+J_{\frac{a+b}{2 a b}-}^{\alpha}(g \circ h)(1 / b)\right]
\end{aligned}
$$

with $\alpha>0$ and $h(x)=1 / x, x \in\left[\frac{1}{b}, \frac{1}{a}\right]$. 
Proof Since $f$ is a harmonically convex function on $[a, b]$, multiplying both sides of (7) by $2 t^{\alpha-1} g\left(\frac{a b}{t b+(1-t) a}\right)$, then integrating the resulting inequality with respect to $t$ over $\left[0, \frac{1}{2}\right]$, we obtain

$$
\begin{aligned}
2 f & \left(\frac{2 a b}{a+b}\right) \int_{0}^{\frac{1}{2}} t^{\alpha-1} g\left(\frac{a b}{t b+(1-t) a}\right) d t \\
\leq & \int_{0}^{\frac{1}{2}} t^{\alpha-1}\left[f\left(\frac{a b}{t a+(1-t) b}\right)+f\left(\frac{a b}{t b+(1-t) a}\right)\right] g\left(\frac{a b}{t b+(1-t) a}\right) d t \\
= & \int_{0}^{\frac{1}{2}} t^{\alpha-1} f\left(\frac{a b}{t a+(1-t) b}\right) g\left(\frac{a b}{t b+(1-t) a}\right) d t \\
& +\int_{0}^{\frac{1}{2}} t^{\alpha-1} f\left(\frac{a b}{t b+(1-t) a}\right) g\left(\frac{a b}{t b+(1-t) a}\right) d t .
\end{aligned}
$$

Since $g$ is harmonically symmetric with respect to $\frac{2 a b}{a+b}$, using Definition 3 we have $g\left(\frac{1}{x}\right)=g\left(\frac{1}{\left(\frac{1}{a}\right)+\left(\frac{1}{b}\right)-x}\right)$, for all $x \in\left[\frac{1}{b}, \frac{1}{a}\right]$. Setting $x=\frac{t b+(1-t) a}{a b}$ and $d x=\left(\frac{b-a}{a b}\right) d t$ gives

$$
\begin{aligned}
& 2\left(\frac{a b}{b-a}\right)^{\alpha} f\left(\frac{2 a b}{a+b}\right) \int_{\frac{1}{b}}^{\frac{a+b}{2 a b}}\left(x-\frac{1}{b}\right)^{\alpha-1} g\left(\frac{1}{x}\right) d x \\
& \leq\left(\frac{a b}{b-a}\right)^{\alpha}\left\{\begin{array}{c}
\int_{\frac{1}{b}}^{\frac{a+b}{2 a b}}\left(x-\frac{1}{b}\right)^{\alpha-1} f\left(\frac{1}{\frac{1}{a}+\frac{1}{b}-x}\right) g\left(\frac{1}{x}\right) d x \\
+\int_{\frac{1}{b}}^{\frac{a+b}{2 a b}}\left(x-\frac{1}{b}\right)^{\alpha-1} f\left(\frac{1}{x}\right) g\left(\frac{1}{x}\right) d x
\end{array}\right\} \\
& =\left(\frac{a b}{b-a}\right)^{\alpha}\left\{\begin{array}{c}
\int_{\frac{a+b}{2 a b}}^{\frac{1}{a}}\left(\frac{1}{a}-x\right)^{\alpha-1} f\left(\frac{1}{x}\right) g\left(\frac{1}{\frac{1}{a}+\frac{1}{b}-x}\right) d x \\
+\int_{\frac{1}{b}}^{\frac{a+b}{2}}\left(x-\frac{1}{b}\right)^{\alpha-1} f\left(\frac{1}{x}\right) g\left(\frac{1}{x}\right) d x
\end{array}\right\} \\
& =\left(\frac{a b}{b-a}\right)^{\alpha}\left\{\begin{array}{c}
\int_{\frac{a+b}{2}}^{\frac{1}{a}}\left(\frac{1}{a}-x\right)^{\alpha-1} f\left(\frac{1}{x}\right) g\left(\frac{1}{x}\right) d x \\
+\int_{\frac{1}{b}}^{\frac{a+b}{2 a b}}\left(x-\frac{1}{b}\right)^{\alpha-1} f\left(\frac{1}{x}\right) g\left(\frac{1}{x}\right) d x
\end{array}\right\} .
\end{aligned}
$$

Therefore, by Lemma 2 we have

$$
\begin{gathered}
\left(\frac{a b}{b-a}\right)^{\alpha} \Gamma(\alpha) f\left(\frac{2 a b}{a+b}\right)\left[J_{\frac{a+b}{2 a b}+}^{\alpha}(g \circ h)(1 / a)+J_{\frac{a+b}{2 a b}-}^{\alpha}(g \circ h)(1 / b)\right] \\
\leq\left(\frac{a b}{b-a}\right)^{\alpha} \Gamma(\alpha)\left[J_{\frac{a+b}{2 a b}+}^{\alpha}(f g \circ h)(1 / a)+J_{\frac{a+b}{2 a b}-}^{\alpha}(f g \circ h)(1 / b)\right]
\end{gathered}
$$

and the first inequality is proved.

For the proof of the second inequality in (9) we first note that if $f$ is a harmonically convex function, then, multiplying both sides of (8) by $t^{\alpha-1} g\left(\frac{a b}{t b+(1-t) a}\right)$ and integrating the resulting inequality with respect to $t$ over $\left[0, \frac{1}{2}\right]$, we obtain 


$$
\begin{aligned}
& \int_{0}^{\frac{1}{2}} t^{\alpha-1} f\left(\frac{a b}{t a+(1-t) b}\right) g\left(\frac{a b}{t b+(1-t) a}\right) d t \\
& \quad+\int_{0}^{\frac{1}{2}} t^{\alpha-1} f\left(\frac{a b}{t b+(1-t) a}\right) g\left(\frac{a b}{t b+(1-t) a}\right) d t \\
& \leq[f(a)+f(b)] \int_{0}^{\frac{1}{2}} t^{\alpha-1} g\left(\frac{a b}{t b+(1-t) a}\right) d t
\end{aligned}
$$

i.e.

$$
\begin{aligned}
& \left(\frac{a b}{b-a}\right)^{\alpha} \Gamma(\alpha)\left[J_{\frac{a+b}{2 a b}+}^{\alpha}(f g \circ h)(1 / a)+J_{\frac{a+b}{2 a b}-}^{\alpha}(f g \circ h)(1 / b)\right] \\
& \quad \leq\left(\frac{a b}{b-a}\right)^{\alpha} \Gamma(\alpha)\left(\frac{f(a)+f(b)}{2}\right)\left[J_{\frac{a+b}{2 a b}+}^{\alpha}(g \circ h)(1 / a)+J_{\frac{a+b}{2 a b}-}^{\alpha}(g \circ h)(1 / b)\right] .
\end{aligned}
$$

The proof is completed.

\section{Remark 1 In Theorem 5,}

(i) if we take $\alpha=1$, then inequality (9) becomes inequality (5) of Theorem 3.

(ii) if we take $g(x)=1$, then inequality (9) becomes inequality (6) of Theorem 4 .

(iii) if we take $\alpha=1$ and $g(x)=1$, then inequality (9) becomes inequality (4) of Theorem 2.

Lemma 3 Let $f: I \subset(0, \infty) \rightarrow \mathbb{R}$ be a differentiable function on $I^{\circ}$, the interior of $I$, such that $f^{\prime} \in L[a, b]$, where $a, b \in I$. If $g:[a, b] \rightarrow \mathbb{R}$ is integrable and harmonically symmetric with respect to $\frac{2 a b}{a+b}$, then the following equality for fractional integrals holds:

$$
\begin{aligned}
f\left(\frac{2 a b}{a+b}\right)\left[J_{\frac{a+b}{2 a b}+}^{\alpha}(g \circ h)(1 / a)+J_{\frac{a+b}{2 a b}-}^{\alpha}(g \circ h)(1 / b)\right] \\
-\quad\left[J_{\frac{a+b}{2 a b}+}^{\alpha}(f g \circ h)(1 / a)+J_{\frac{a+b}{2 a b}-}^{\alpha}(f \circ \circ h)(1 / b)\right] \\
=\frac{1}{\Gamma(\alpha)}\left[\begin{array}{c}
\int_{\frac{1}{b}}^{\frac{a+b}{2 a b}}\left(\int_{\frac{1}{b}}^{t}\left(s-\frac{1}{b}\right)^{\alpha-1}(g \circ h)(s) d s\right)(f \circ h)^{\prime}(t) d t \\
-\int_{\frac{a+b}{2 a b}}^{\frac{1}{a}}\left(\int_{t}^{\frac{1}{a}}\left(\frac{1}{a}-s\right)^{\alpha-1}(g \circ h)(s) d s\right)(f \circ h)^{\prime}(t) d t
\end{array}\right]
\end{aligned}
$$

with $\alpha>0$ and $h(x)=1 / x, x \in\left[\frac{1}{b}, \frac{1}{a}\right]$.

Proof It suffices to note that

$$
\begin{aligned}
I= & \int_{\frac{1}{b}}^{\frac{a+b}{2 a b}}\left(\int_{\frac{1}{b}}^{t}\left(s-\frac{1}{b}\right)^{\alpha-1}(g \circ h)(s) d s\right)(f \circ h)^{\prime}(t) d t \\
& -\int_{\frac{a+b}{2 a b}}^{\frac{1}{a}}\left(\int_{t}^{\frac{1}{a}}\left(\frac{1}{a}-s\right)^{\alpha-1}(g \circ h)(s) d s\right)(f \circ h)^{\prime}(t) d t \\
= & I_{1}-I_{2} .
\end{aligned}
$$


By integration by parts and Lemma 2 we get

$$
\begin{aligned}
& I_{1}=\left.\left(\int_{\frac{1}{b}}^{t}\left(s-\frac{1}{b}\right)^{\alpha-1}(g \circ h)(s) d s\right)(f \circ h)(t)\right|_{\frac{1}{b}} ^{\frac{a+b}{2 a b}} \\
&-\int_{\frac{1}{b}}^{\frac{a+b}{2 a b}}\left(t-\frac{1}{b}\right)^{\alpha-1}(g \circ h)(t)(f \circ h)(t) d t \\
&=\left(\int_{\frac{1}{b}}^{\frac{a+b}{2 a b}}\left(s-\frac{1}{b}\right)^{\alpha-1}(g \circ h)(s) d s\right) f\left(\frac{2 a b}{a+b}\right) \\
&-\int_{\frac{1}{b}}^{\frac{a+b}{2 a b}}\left(t-\frac{1}{b}\right)^{\alpha-1}(g \circ h)(t)(f \circ h)(t) d t \\
&= \Gamma(\alpha)\left[f\left(\frac{2 a b}{a+b}\right) J_{\frac{a+b}{2 a b}-}^{\alpha}(g \circ h)(1 / b)-J_{\frac{a+b}{2 a b}-}^{\alpha}(f g \circ h)(1 / b)\right] \\
&= \Gamma(\alpha)\left[\frac{f\left(\frac{2 a b}{a+b}\right)}{2}\left[J_{\frac{a+b}{2 a b}+}^{\alpha}(g \circ h)(1 / a)+J_{\frac{a+b}{2 a b}-}^{\alpha}(g \circ h)(1 / b)\right]\right] \\
&-J_{\frac{a+b}{2 a b}-\alpha}^{\alpha}(f g \circ h)(1 / b)
\end{aligned}
$$

and similarly

$$
\begin{aligned}
& I_{2}=\left.\left(\int_{t}^{\frac{1}{a}}\left(\frac{1}{a}-s\right)^{\alpha-1}(g \circ h)(s) d s\right)(f \circ h)(t)\right|_{\frac{a+b}{2 a b}} ^{\frac{1}{a}} \\
&+\int_{\frac{a+b}{2 a b}}^{\frac{1}{a}}\left(\frac{1}{a}-t\right)^{\alpha-1}(g \circ h)(t)(f \circ h)(t) d t \\
&=-\left(\int_{\frac{a+b}{2 a b}}^{\frac{1}{a}}\left(\frac{1}{a}-s\right)^{\alpha-1}(g \circ h)(s) d s\right) f\left(\frac{2 a b}{a+b}\right) \\
&+\int_{\frac{a+b}{2 a b}}^{\frac{1}{a}}\left(\frac{1}{a}-t\right)^{\alpha-1}(g \circ h)(t)(f \circ h)(t) d t \\
&= \Gamma(\alpha)\left[-f\left(\frac{2 a b}{a+b}\right) J_{\frac{a+b}{2 a b}+}^{\alpha}(g \circ h)(1 / a)+J_{\frac{a+b}{2 a b}+}^{\alpha}(f g \circ h)(1 / a)\right] \\
&= \Gamma(\alpha)\left[-\frac{f\left(\frac{2 a b}{a+b}\right)}{2}\left[J_{\frac{a+b}{2 a b}+}^{\alpha}(g \circ h)(1 / a)+J_{\frac{a+b}{2 a b}-}^{\alpha}(g \circ h)(1 / b)\right]\right] . \\
&+J_{\frac{a+b}{2 a b}+}^{\alpha}(f g \circ h)(1 / a)
\end{aligned}
$$

Thus, we can write

$$
I=I_{1}-I_{2}=\Gamma(\alpha)\left\{\begin{array}{l}
f\left(\frac{2 a b}{a+b}\right)\left[J_{\frac{a+b}{2 a b}+}^{\alpha}(g \circ h)(1 / a)+J_{\frac{a+b}{2 a b}-}^{\alpha}(g \circ h)(1 / b)\right] \\
-\left[J_{\frac{a+b}{2 a b}+}^{\alpha}(f g \circ h)(1 / a)+J_{\frac{a+b}{2 a b}-}^{\alpha}(f g \circ h)(1 / b)\right]
\end{array}\right\}
$$

Multiplying both sides by $(\Gamma(\alpha))^{-1}$ we obtain (10). This completes the proof. 
Theorem 6 Let $f: I \subset(0, \infty) \rightarrow \mathbb{R}$ be a differentiable function on $I^{\circ}$, the interior of $I$, such that $f^{\prime} \in L[a, b]$, where $a, b \in I$ and $a<b$. If $\left|f^{\prime}\right|$ is harmonically convex on $[a, b]$, $g:[a, b] \rightarrow \mathbb{R}$ is continuous and harmonically symmetric with respect to $\frac{2 a b}{a+b}$, then the following inequality for fractional integrals holds:

$$
\begin{aligned}
& \left|\begin{array}{c}
f\left(\frac{2 a b}{a+b}\right)\left[J_{\frac{a+b}{2 a b}+}^{\alpha}(g \circ h)(1 / a)+J_{\frac{a+b}{2 a b}-}^{\alpha}(g \circ h)(1 / b)\right] \\
-\left[J_{\frac{a+b}{2 a b}+}^{\alpha}(f g \circ h)(1 / a)+J_{\frac{a+b}{2 a b}-}^{\alpha}(f g \circ h)(1 / b)\right]
\end{array}\right| \\
& \quad \leq \frac{\|g\|_{\infty} a b(b-a)}{\Gamma(\alpha+1)}\left(\frac{b-a}{a b}\right)^{\alpha}\left[C_{1}(\alpha)\left|f^{\prime}(a)\right|+C_{2}(\alpha)\left|f^{\prime}(b)\right|\right]
\end{aligned}
$$

where

$$
\begin{aligned}
& C_{1}(\alpha)=\left[\begin{array}{c}
\frac{b^{-2}}{(\alpha+1)(\alpha+2)}{ }_{2} F_{1}\left(2, \alpha+1 ; \alpha+3 ; 1-\frac{a}{b}\right) \\
-\frac{(a+b)^{-2}}{(\alpha+1)(\alpha+2)}{ }_{2} F_{1}\left(2, \alpha+1 ; \alpha+3 ; \frac{b-a}{b+a}\right)
\end{array}\right], \\
& C_{2}(\alpha)=\left[\begin{array}{c}
\frac{b^{-2}}{\alpha+2}{ }_{2} F_{1}\left(2, \alpha+2 ; \alpha+3 ; 1-\frac{a}{b}\right) \\
-\frac{2(a+b)^{-2}}{\alpha+1}{ }_{2} F_{1}\left(2, \alpha+1 ; \alpha+2 ; \frac{b-a}{b+a}\right) \\
+\frac{(a+b))^{-2}}{(\alpha+1)(\alpha+2)} 2{ }_{2} F_{1}\left(2, \alpha+1 ; \alpha+3 ; \frac{b-a}{b+a}\right)
\end{array}\right],
\end{aligned}
$$

with $0<\alpha \leq 1$ and $h(x)=1 / x, x \in\left[\frac{1}{b}, \frac{1}{a}\right]$.

Proof From Lemma 3 we have

$$
\begin{aligned}
& \left|\begin{array}{r}
f\left(\frac{2 a b}{a+b}\right)\left[J_{\frac{a+b}{2 a b}+}^{\alpha}(g \circ h)(1 / a)+J_{\frac{a+b}{2 a b}-}^{\alpha}(g \circ h)(1 / b)\right] \\
-\left[J_{\frac{a+b}{2 a b}+}^{\alpha}(f g \circ h)(1 / a)+J_{\frac{a+b}{2 a b}-}^{\alpha}(f g \circ h)(1 / b)\right]
\end{array}\right| \\
& \leq \frac{1}{\Gamma(\alpha)}\left[\begin{array}{c}
\int_{\frac{1}{b}}^{\frac{a+b}{2 a b}}\left(\int_{\frac{1}{b}}^{t}\left(s-\frac{1}{b}\right)^{\alpha-1}|(g \circ h)(s)| d s\right)\left|(f \circ h)^{\prime}(t)\right| d t \\
\left.+\int_{\frac{a+b}{2 a b}}^{\frac{1}{a}}\left(\int_{t}^{\frac{1}{a}}\left(\frac{1}{a}-s\right)^{\alpha-1}|(g \circ h)(s)| d s\right)\left|(f \circ h)^{\prime}(t)\right| d t\right]
\end{array}\right. \\
& \leq \frac{\|g\|_{\infty}}{\Gamma(\alpha)}\left[\begin{array}{c}
\int_{\frac{1}{b}}^{\frac{a+b}{2 a b}}\left(\int_{\frac{1}{b}}^{t}\left(s-\frac{1}{b}\right)^{\alpha-1} d s\right)\left|(f \circ h)^{\prime}(t)\right| d t \\
\left.+\int_{\frac{a+b}{2 a b}}^{\frac{1}{a}}\left(\int_{t}^{\frac{1}{a}}\left(\frac{1}{a}-s\right)^{\alpha-1} d s\right)\left|(f \circ h)^{\prime}(t)\right| d t\right]
\end{array}\right. \\
& =\frac{\|g\|_{\infty}}{\Gamma(\alpha)}\left[\begin{array}{c}
\int_{\frac{1}{b}}^{\frac{a+b}{2 a b}} \frac{\left(t-\frac{1}{b}\right)^{\alpha}}{\alpha} \frac{1}{t^{2}}\left|f^{\prime}\left(\frac{1}{t}\right)\right| d t \\
\left.+\int_{\frac{a+b}{2 a b}}^{\frac{1}{a}} \frac{\left(\frac{1}{a}-t\right)^{\alpha}}{\alpha} \frac{1}{t^{2}}\left|f^{\prime}\left(\frac{1}{t}\right)\right| d t\right] .
\end{array}\right.
\end{aligned}
$$


Setting $t=\frac{u b+(1-u) a}{a b}$ and $d t=\left(\frac{b-a}{a b}\right) d u$ gives

$$
\begin{aligned}
& \left|f\left(\frac{2 a b}{a+b}\right)\left[J_{\frac{a+b}{2 a b}+}^{\alpha}(g \circ h)(1 / a)+J_{\frac{a+b}{2 a b}-}^{\alpha}(g \circ h)(1 / b)\right]\right| \\
& \quad-\left[J_{\frac{a+b}{2 a b}+}^{\alpha}(f g \circ h)(1 / a)+J_{\frac{a+b}{2 a b}-\alpha}^{\alpha}(f g \circ h)(1 / b)\right] \\
& \quad \leq \frac{\|g\|_{\infty} a b(b-a)}{\Gamma(\alpha+1)}\left(\frac{b-a}{a b}\right)^{\alpha}\left[\begin{array}{c}
\int_{0}^{\frac{1}{2}} \frac{u^{\alpha}}{(u b+(1-u) a)^{2}}\left|f^{\prime}\left(\frac{a b}{u b+(1-u) a}\right)\right| d u \\
+\int_{\frac{1}{2}}^{1} \frac{(1-u)^{\alpha}}{(u b+(1-u) a)^{2}}\left|f^{\prime}\left(\frac{a b}{u b+(1-u) a}\right)\right| d u
\end{array}\right] .
\end{aligned}
$$

Since $\left|f^{\prime}\right|$ is harmonically convex on $[a, b]$, we have

$$
\left|f^{\prime}\left(\frac{a b}{u b+(1-u) a t}\right)\right| \leq u\left|f^{\prime}(a)\right|+(1-u)\left|f^{\prime}(b)\right| .
$$

If we use (13) in (12), we have

$$
\begin{aligned}
& f\left(\frac{2 a b}{a+b}\right)\left[J_{\frac{a+b}{2 a b}+}^{\alpha}(g \circ h)(1 / a)+J_{\frac{a+b}{2 a b}-}^{\alpha}(g \circ h)(1 / b)\right] \\
& -\left[J_{\frac{a+b}{2 a b}+}^{\alpha}(f g \circ h)(1 / a)+J_{\frac{a+b}{2 a b}-}^{\alpha}(f g \circ h)(1 / b)\right] \\
& \leq \frac{\|g\|_{\infty} a b(b-a)}{\Gamma(\alpha+1)}\left(\frac{b-a}{a b}\right)^{\alpha} \\
& \times\left[\begin{array}{c}
\int_{0}^{\frac{1}{2}} \frac{u^{\alpha}}{(u b+(1-u) a)^{2}}\left[u\left|f^{\prime}(a)\right|+(1-u)\left|f^{\prime}(b)\right|\right] d u \\
+\int_{\frac{1}{2}}^{1} \frac{(1-u)^{\alpha}}{(u b+(1-u) a)^{2}}\left[u\left|f^{\prime}(a)\right|+(1-u)\left|f^{\prime}(b)\right|\right] d u
\end{array}\right] .
\end{aligned}
$$

Calculating the following integrals by Lemma 1, we have

$$
\begin{aligned}
& \int_{0}^{\frac{1}{2}} \frac{u^{\alpha+1}}{(u b+(1-u) a)^{2}} d u+\int_{\frac{1}{2}}^{1} \frac{(1-u)^{\alpha} u}{(u b+(1-u) a)^{2}} d u \\
&= \int_{0}^{1} \frac{(1-u)^{\alpha} u}{(u b+(1-u) a)^{2}} d u-\int_{0}^{\frac{1}{2}} \frac{(1-u)^{\alpha}-u^{\alpha}}{(u b+(1-u) a)^{2}} u d u \\
& \leq \int_{0}^{1} \frac{(1-u)^{\alpha} u}{(u b+(1-u) a)^{2}} d u-\int_{0}^{\frac{1}{2}} \frac{(1-2 u)^{\alpha}}{(u b+(1-u) a)^{2}} u d u \\
&= \int_{0}^{1} \frac{(1-u)^{\alpha} u}{(u b+(1-u) a)^{2}} d u-\frac{1}{4} \int_{0}^{1} \frac{(1-u)^{\alpha}}{\left(\frac{u}{2} b+\left(1-\frac{u}{2}\right) a\right)^{2}} u d u \\
&= \int_{0}^{1}(1-u) u^{\alpha} b^{-2}\left(1-u\left(1-\frac{a}{b}\right)\right)^{-2} d u \\
&-\frac{1}{4} \int_{0}^{1}(1-v) v^{\alpha}\left(\frac{a+b}{2}\right)^{-2}\left(1-v\left(\frac{b-a}{b+a}\right)\right)^{-2} d v \\
&= {\left[\frac{b^{-2}}{(\alpha+1)(\alpha+2)}{ }_{2} F_{1}\left(2, \alpha+1 ; \alpha+3 ; 1-\frac{a}{b}\right)\right] } \\
&\left.-\frac{(a+b)^{-2}}{(\alpha+1)(\alpha+2)} 2 F_{1}\left(2, \alpha+1 ; \alpha+3 ; \frac{b-a}{b+a}\right)\right] \\
&= C_{1}(\alpha)
\end{aligned}
$$


and similarly we get

$$
\begin{aligned}
\int_{0}^{\frac{1}{2}} & \frac{u^{\alpha}}{(u b+(1-u) a)^{2}}(1-u) d u+\int_{\frac{1}{2}}^{1} \frac{(1-u)^{\alpha}}{(u b+(1-u) a)^{2}}(1-u) d u \\
= & \int_{0}^{1} \frac{(1-u)^{\alpha+1}}{(u b+(1-u) a)^{2}} d u-\int_{0}^{\frac{1}{2}} \frac{(1-u)^{\alpha}-u^{\alpha}}{(u b+(1-u) a)^{2}}(1-u) d u \\
\leq & \int_{0}^{1} \frac{(1-u)^{\alpha+1}}{(u b+(1-u) a)^{2}} d u-\int_{0}^{\frac{1}{2}} \frac{(1-2 u)^{\alpha}}{(u b+(1-u) a)^{2}}(1-u) d u \\
= & \int_{0}^{1} \frac{(1-u)^{\alpha+1}}{(u b+(1-u) a)^{2}} d u-\int_{0}^{\frac{1}{2}} \frac{(1-2 u)^{\alpha}}{(u b+(1-u) a)^{2}} d u \\
& +\int_{0}^{\frac{1}{2}} \frac{u(1-2 u)^{\alpha}}{(u b+(1-u) a)^{2}} d u \\
= & \int_{0}^{1} \frac{u^{\alpha+1}}{(u a+(1-u) b)^{2}} d u-\frac{1}{2} \int_{0}^{1} \frac{(1-u)^{\alpha}}{\left(\frac{u}{2} b+\left(1-\frac{u}{2}\right) a\right)^{2}} d u \\
& +\frac{1}{4} \int_{0}^{1} \frac{u(1-u)^{\alpha}}{\left(\frac{u}{2} b+\left(1-\frac{u}{2}\right) a\right)^{2}} d u \\
= & \int_{0}^{1} \frac{u^{\alpha+1}}{(u a+(1-u) b)^{2}} d u-\frac{1}{2} \int_{0}^{1} v^{\alpha}\left(\frac{a+b}{2}\right)^{-2}\left(1-v\left(\frac{b-a}{b+a}\right)\right)^{-2} d v \\
& +\frac{1}{4} \int_{0}^{1}(1-v) v^{\alpha}\left(\frac{a+b}{2}\right)^{-2}\left(1-v\left(\frac{b-a}{b+a}\right)\right)^{-2} d v \\
= & {\left[\begin{array}{l}
\frac{b^{-2}}{\alpha+2}{ }_{2} F_{1}\left(2, \alpha+2 ; \alpha+3 ; 1-\frac{a}{b}\right) \\
-\frac{2(a+b)^{-2}}{\alpha+1}{ }_{2} F_{1}\left(2, \alpha+1 ; \alpha+2 ; \frac{b-a}{b+a}\right) \\
+\frac{(a+b)^{-2}}{(\alpha+1)(\alpha+2)}{ }_{2} F_{1}\left(2, \alpha+1 ; \alpha+3 ; \frac{b-a}{b+a}\right)
\end{array}\right] } \\
= & C_{2}(\alpha) .
\end{aligned}
$$

If we use (15) and (16) in (14), we have (11). This completes the proof.

\section{Corollary 1 In Theorem 6:}

(1) If we take $\alpha=1$ we have the following Hermite-Hadamard-Fejer inequality for harmonically convex functions which is related to the left-hand side of (5):

$$
\begin{aligned}
& \left|f\left(\frac{2 a b}{a+b}\right) \int_{a}^{b} \frac{g(x)}{x^{2}} d x-\int_{a}^{b} \frac{f(x) g(x)}{x^{2}} d x\right| \\
& \quad \leq\|g\|_{\infty}(b-a)^{2}\left[C_{1}(1)\left|f^{\prime}(a)\right|+C_{2}(1)\left|f^{\prime}(b)\right|\right],
\end{aligned}
$$

(2) If we take $g(x)=1$ we have following Hermite-Hadamard type inequality for harmonically convex functions in fractional integral forms which is related to the left-hand side of (6):

$$
\begin{aligned}
& \left|f\left(\frac{2 a b}{a+b}\right)-\frac{\Gamma(\alpha+1)}{2^{1-\alpha}}\left(\frac{a b}{b-a}\right)^{\alpha}\left\{\begin{array}{c}
J_{\frac{a+b}{2 a b}+}^{\alpha}(f \circ h)(1 / a) \\
+J_{\frac{a+b}{2 a b}-}^{\alpha}(f \circ h)(1 / b)
\end{array}\right\}\right| \\
& \quad \leq \frac{a b(b-a)}{2^{1-\alpha}}\left[C_{1}(\alpha)\left|f^{\prime}(a)\right|+C_{2}(\alpha)\left|f^{\prime}(b)\right|\right],
\end{aligned}
$$


(3) If we take $\alpha=1$ and $g(x)=1$ we have the following Hermite-Hadamard type inequality for harmonically convex functions which is related to the left-hand side of (4):

$$
\left|f\left(\frac{2 a b}{a+b}\right)-\frac{a b}{b-a} \int_{a}^{b} \frac{f(x)}{x^{2}} d x\right| \leq a b(b-a)\left[C_{1}(1)\left|f^{\prime}(a)\right|+C_{2}(1)\left|f^{\prime}(b)\right|\right] .
$$

Theorem 7 Let $f: I \subset(0, \infty) \rightarrow \mathbb{R}$ be a differentiable function on $I^{\circ}$, the interior of I, such that $f^{\prime} \in L[a, b]$, where $a, b \in I$. If $\left|f^{\prime}\right|^{q}, q \geq 1$, is harmonically convex on $[a, b]$, $g:[a, b] \rightarrow \mathbb{R}$ is continuous and harmonically symmetric with respect to $\frac{2 a b}{a+b}$, then the following inequality for fractional integrals holds:

$$
\begin{gathered}
\left|\begin{array}{c}
\frac{f(a)+f(b)}{2}\left[J_{1 / b+}^{\alpha}(g \circ h)(1 / a)+J_{1 / a-}^{\alpha}(g \circ h)(1 / b)\right] \\
-\left[J_{1 / b+}^{\alpha}(g \circ h)(1 / a)+J_{1 / a-}^{\alpha}(f g \circ h)(1 / b)\right]
\end{array}\right| \\
\leq \frac{\|g\|_{\infty} a b(b-a)}{\Gamma(\alpha+1)}\left(\frac{b-a}{a b}\right)^{\alpha} \\
\quad \times\left[\begin{array}{c}
C_{3}^{1-\frac{1}{q}}(\alpha)\left[\left(\begin{array}{c}
C_{4}(\alpha)\left|f^{\prime}(a)\right|^{q} \\
+C_{5}(\alpha)\left|f^{\prime}(b)\right|^{q}
\end{array}\right)\right]^{\frac{1}{q}} \\
+C_{6}^{1-\frac{1}{q}}(\alpha)\left[\left(\begin{array}{c}
C_{7}(\alpha)\left|f^{\prime}(a)\right|^{q} \\
+C_{8}(\alpha)\left|f^{\prime}(b)\right|^{q}
\end{array}\right)\right]^{\frac{1}{q}}
\end{array}\right]
\end{gathered}
$$

where

$$
\begin{aligned}
& C_{3}(\alpha)=\frac{(a+b)^{-2}}{2^{\alpha-1}(\alpha+1)}{ }_{2} F_{1}\left(2,1 ; \alpha+2 ; \frac{b-a}{b+a}\right), \\
& C_{4}(\alpha)=\frac{(a+b)^{-2}}{2^{\alpha}(\alpha+2)}{ }_{2} F_{1}\left(2,1 ; \alpha+3 ; \frac{b-a}{b+a}\right), \\
& C_{5}(\alpha)=C_{3}(\alpha)-C_{4}(\alpha), \\
& C_{6}(\alpha)=\frac{b^{-2}}{2^{\alpha+1}(\alpha+1)}{ }_{2} F_{1}\left(2, \alpha+1 ; \alpha+2 ; \frac{1}{2}\left(1-\frac{a}{b}\right)\right), \\
& C_{7}(\alpha)=\left[\begin{array}{c}
\frac{b^{-2}}{2^{\alpha+1}(\alpha+1)}{ }_{2} F_{1}\left(2, \alpha+1 ; \alpha+2 ; \frac{1}{2}\left(1-\frac{a}{b}\right)\right) \\
-\frac{b^{-2}}{2^{\alpha+2}(\alpha+2)}{ }_{2} F_{1}\left(2, \alpha+2 ; \alpha+3 ; \frac{1}{2}\left(1-\frac{a}{b}\right)\right)
\end{array}\right], \\
& C_{8}(\alpha)=C_{6}(\alpha)-C_{7}(\alpha), \\
& \text { with } \alpha>1 \text { and } h(x)=1 / x, x \in\left[\frac{1}{b}, \frac{1}{a}\right] .
\end{aligned}
$$

Proof Using (12), power mean inequality and the harmonically convexity of $\left|f^{\prime}\right|^{q}$, it follows that 


$$
\begin{aligned}
& \left|\begin{array}{c}
f\left(\frac{2 a b}{a+b}\right)\left[J_{\frac{a+b}{2 a b}+}^{\alpha}(g \circ h)(1 / a)+J_{\frac{a+b}{2 a b}-}^{\alpha}(g \circ h)(1 / b)\right] \\
-\left[J_{\frac{a+b}{2 a b}+}^{\alpha}(f g \circ h)(1 / a)+J_{\frac{a+b}{2 a b}-}^{\alpha}(f g \circ h)(1 / b)\right]
\end{array}\right| \\
& \leq \frac{\|g\|_{\infty} a b(b-a)}{\Gamma(\alpha+1)}\left(\frac{b-a}{a b}\right)^{\alpha}\left[\begin{array}{c}
\int_{0}^{\frac{1}{2}} \frac{u^{\alpha}}{(u b+(1-u) a)^{2}}\left|f^{\prime}\left(\frac{a b}{u b+(1-u) a}\right)\right| d u \\
+\int_{\frac{1}{2}}^{1} \frac{(1-u)^{2}}{(u b+(1-u) a)^{2}}\left|f^{\prime}\left(\frac{a b}{u b+(1-u) a}\right)\right| d u
\end{array}\right] \\
& \leq \frac{\|g\|_{\infty} a b(b-a)}{\Gamma(\alpha+1)}\left(\frac{b-a}{a b}\right)^{\alpha} \\
& {\left[\begin{array}{c}
\left(\int_{0}^{\frac{1}{2}} \frac{u^{\alpha}}{(u b+(1-u) a)^{2}} d u\right)^{1-\frac{1}{q}} \\
\times\left(\int_{0}^{\frac{1}{2}} \frac{u^{\alpha}}{(u b+(1-u) a)^{2}}\left|f^{\prime}\left(\frac{a b}{u b+(1-u) a}\right)\right|^{q} d u\right)^{\frac{1}{q}} \\
+\left(\int_{\frac{1}{2}}^{1} \frac{(1-u)^{\alpha}}{(u b+(1-u) a)^{2}} d u\right)^{1-\frac{1}{q}} \\
\times\left(\int_{\frac{1}{2}}^{1} \frac{(1-u)^{\alpha}}{(u b+(1-u) a)^{2}}\left|f^{\prime}\left(\frac{a b}{u b+(1-u) a}\right)\right|^{q} d u\right)^{\frac{1}{q}}
\end{array}\right]} \\
& \leq \frac{\|g\|_{\infty} a b(b-a)}{\Gamma(\alpha+1)}\left(\frac{b-a}{a b}\right)^{\alpha} \\
& \times\left[\begin{array}{c}
\left(\int_{0}^{\frac{1}{2}} \frac{u^{\alpha}}{(u b+(1-u) a)^{2}} d u\right)^{1-\frac{1}{q}} \\
\times\left(\int_{0}^{\frac{1}{2}} \frac{u^{\alpha}}{(u b+(1-u) a)^{2}}\left[u\left|f^{\prime}(a)\right|^{q}+(1-u)\left|f^{\prime}(b)\right|^{q}\right] d u\right)^{\frac{1}{q}} \\
+\left(\int_{\frac{1}{2}}^{1} \frac{(1-u)^{\alpha}}{(u b+(1-u) a)^{2}} d u\right)^{1-\frac{1}{q}} \\
\times\left(\int_{\frac{1}{2}}^{1} \frac{(1-u)^{\alpha}}{(u b+(1-u) a)^{2}}\left[u\left|f^{\prime}(a)\right|^{q}+(1-u)\left|f^{\prime}(b)\right|^{q}\right] d u\right)^{\frac{1}{q}}
\end{array}\right] \\
& =\frac{\|g\|_{\infty} a b(b-a)}{\Gamma(\alpha+1)}\left(\frac{b-a}{a b}\right)^{\alpha} \\
& \times\left[\begin{array}{c}
\left(\int_{0}^{\frac{1}{2}} \frac{u^{\alpha}}{(u b+(1-u) a)^{2}} d u\right)^{1-\frac{1}{q}} \\
\times\left(\begin{array}{c}
\int_{0}^{\frac{1}{2}} \frac{u^{\alpha+1}}{(u b+(1-u) a)^{2}} d u\left|f^{\prime}(a)\right|^{q} \\
+\left.\left.\int_{0}^{\frac{1}{2}} \frac{u^{\alpha}}{(u b+(1-u) a)^{2}}(1-u) d u\right|^{\prime}(b)\right|^{q} \\
+\left(\int_{\frac{1}{2}}^{1} \frac{(1-u)^{\alpha}}{(u b+(1-u) a)^{2}} d u\right)^{1-\frac{1}{q}} \\
\times\left(\int_{\frac{1}{2}}^{1} \frac{(1-u)^{\alpha}}{(u b+(1-u) a)^{2}} u d u\left|f^{\prime}(a)\right|^{q}\right. \\
+\int_{\frac{1}{2}}^{1} \frac{(1-u)^{\alpha+1}}{(u b+(1-u) a)^{2}} d u\left|f^{\prime}(b)\right|^{q}
\end{array}\right)^{\frac{1}{q}}
\end{array}\right] .
\end{aligned}
$$

For the appearing integrals, we have

$$
\begin{aligned}
\int_{0}^{\frac{1}{2}} \frac{u^{\alpha}}{(u b+(1-u) a)^{2}} d u & =\frac{1}{2^{\alpha+1}} \int_{0}^{1} \frac{u^{\alpha}}{\left(\frac{u}{2} b+\left(1-\frac{u}{2}\right) a\right)^{2}} d u \\
& =\frac{1}{2^{\alpha+1}} \int_{0}^{1}(1-v)^{\alpha}\left(\frac{a+b}{2}\right)^{-2}\left(1-v\left(\frac{b-a}{b+a}\right)\right)^{-2} d u \\
& =\frac{(a+b)^{-2}}{2^{\alpha-1}(\alpha+1)}{ }_{2} F_{1}\left(2,1 ; \alpha+2 ; \frac{b-a}{b+a}\right) \\
& =C_{3}(\alpha)
\end{aligned}
$$




$$
\begin{aligned}
\int_{0}^{\frac{1}{2}} \frac{u^{\alpha+1}}{(u b+(1-u) a)^{2}} d u & =\frac{1}{2^{\alpha+2}} \int_{0}^{1} \frac{u^{\alpha+1}}{\left(\frac{u}{2} b+\left(1-\frac{u}{2}\right) a\right)^{2}} d u \\
& =\frac{1}{2^{\alpha+2}} \int_{0}^{1}(1-v)^{\alpha+1}\left(\frac{a+b}{2}\right)^{-2}\left(1-v\left(\frac{b-a}{b+a}\right)\right)^{-2} d u \\
& =\frac{(a+b)^{-2}}{2^{\alpha}(\alpha+2)}{ }_{2} F_{1}\left(2,1 ; \alpha+3 ; \frac{b-a}{b+a}\right) \\
& =C_{4}(\alpha)
\end{aligned}
$$

$$
\int_{0}^{\frac{1}{2}} \frac{u^{\alpha}}{(u b+(1-u) a)^{2}}(1-u) d u=C_{3}(\alpha)-C_{4}(\alpha)=C_{5}(\alpha)
$$

$$
\begin{aligned}
\int_{\frac{1}{2}}^{1} \frac{(1-u)^{\alpha}}{(u b+(1-u) a)^{2}} d u & =\int_{0}^{\frac{1}{2}} \frac{u^{\alpha}}{(u a+(1-u) b)^{2}} d u \\
& =\frac{1}{2^{\alpha+1}} \int_{0}^{1} \frac{u^{\alpha}}{\left(\frac{u}{2} a+\left(1-\frac{u}{2}\right) b\right)^{2}} d u \\
& =\frac{1}{2^{\alpha+1}} \int_{0}^{1} u^{\alpha} b^{-2}\left(1-\frac{u}{2}\left(1-\frac{a}{b}\right)\right)^{-2} d u \\
& =\frac{b^{-2}}{2^{\alpha+1}(\alpha+1)}{ }_{2} F_{1}\left(2, \alpha+1 ; \alpha+2 ; \frac{1}{2}\left(1-\frac{a}{b}\right)\right) \\
& =C_{6}(\alpha),
\end{aligned}
$$

$$
\begin{aligned}
& \int_{\frac{1}{2}}^{1} \frac{(1-u)^{\alpha}}{(u b+(1-u) a)^{2}} u d u=\int_{0}^{\frac{1}{2}} \frac{u^{\alpha}(1-u)}{(u a+(1-u) b)^{2}} d u \\
& =\int_{0}^{\frac{1}{2}} \frac{u^{\alpha}}{(u a+(1-u) b)^{2}} d u-\int_{0}^{\frac{1}{2}} \frac{u^{\alpha+1}}{(u a+(1-u) b)^{2}} d u \\
& =\frac{1}{2^{\alpha+1}} \int_{0}^{1} \frac{u^{\alpha}}{\left(\frac{u}{2} a+\left(1-\frac{u}{2}\right) b\right)^{2}} d u \\
& -\frac{1}{2^{\alpha+2}} \int_{0}^{1} \frac{u^{\alpha+1}}{\left(\frac{u}{2} a+\left(1-\frac{u}{2}\right) b\right)^{2}} d u \\
& =\frac{1}{2^{\alpha+1}} \int_{0}^{1} u^{\alpha} b^{-2}\left(1-\frac{u}{2}\left(1-\frac{a}{b}\right)\right)^{-2} d u \\
& -\frac{1}{2^{\alpha+2}} \int_{0}^{1} u^{\alpha+1} b^{-2}\left(1-\frac{u}{2}\left(1-\frac{a}{b}\right)\right)^{-2} d u \\
& =\left[\begin{array}{c}
\frac{b^{-2}}{2^{\alpha+1}(\alpha+1)}{ }_{2} F_{1}\left(2, \alpha+1 ; \alpha+2 ; \frac{1}{2}\left(1-\frac{a}{b}\right)\right) \\
-\frac{b^{-2}}{2^{\alpha+2}(\alpha+2)}{ }_{2} F_{1}\left(2, \alpha+2 ; \alpha+3 ; \frac{1}{2}\left(1-\frac{a}{b}\right)\right)
\end{array}\right] \\
& =C_{7}(\alpha) \text {, } \\
& \int_{\frac{1}{2}}^{1} \frac{(1-u)^{\alpha+1}}{(u b+(1-u) a)^{2}} d u=\int_{\frac{1}{2}}^{1} \frac{(1-u)^{\alpha}}{(u b+(1-u) a)^{2}} d u-\int_{\frac{1}{2}}^{1} \frac{(1-u)^{\alpha}}{(u b+(1-u) a)^{2}} u d u \\
& =C_{6}(\alpha)-C_{7}(\alpha)=C_{8}(\alpha) \text {. }
\end{aligned}
$$

If we use (19-24) in (18), we have (17). This completes the proof. 


\section{Corollary 2 In Theorem 7:}

(1) If we take $\alpha=1$ we have the following Hermite-Hadamard-Fejer inequality for harmonically convex functions which is related to the left-hand side of (5):

$$
\begin{aligned}
& \left|f\left(\frac{2 a b}{a+b}\right) \int_{a}^{b} \frac{g(x)}{x^{2}} d x-\int_{a}^{b} \frac{f(x) g(x)}{x^{2}} d x\right| \\
& \quad \leq\|g\|_{\infty}(b-a)^{2}\left[\begin{array}{c}
C_{3}^{1-\frac{1}{q}}(1)\left[\left(\begin{array}{c}
C_{4}(1)\left|f^{\prime}(a)\right|^{q} \\
+C_{5}(1)\left|f^{\prime}(b)\right|^{q}
\end{array}\right)\right]^{\frac{1}{q}} \\
+C_{6}^{1-\frac{1}{q}}(1)\left[\left(\begin{array}{c}
C_{7}(1)\left|f^{\prime}(a)\right|^{q} \\
+C_{8}(1)\left|f^{\prime}(b)\right|^{q}
\end{array}\right)\right]^{\frac{1}{q}}
\end{array}\right],
\end{aligned}
$$

(2) If we take $g(x)=1$ we have the following Hermite-Hadamard type inequality for harmonically convex functions in fractional integral forms which is related to the left-hand side of (6):

$$
\begin{gathered}
\left|f\left(\frac{2 a b}{a+b}\right)-\frac{\Gamma(\alpha+1)}{2^{1-\alpha}}\left(\frac{a b}{b-a}\right)^{\alpha}\left\{\begin{array}{c}
J_{\frac{a+b}{2 a b}+}^{\alpha}(f \circ h)(1 / a) \\
+J_{\frac{a+b}{2 a b}-}^{\alpha}(f \circ h)(1 / b)
\end{array}\right\}\right| \\
\leq \frac{a b(b-a)}{2^{1-\alpha}}\left[\begin{array}{c}
\left.C_{3}^{1-\frac{1}{q}}(\alpha)\left[\left(\begin{array}{c}
C_{4}(\alpha)\left|f^{\prime}(a)\right|^{q} \\
+C_{5}(\alpha)\left|f^{\prime}(b)\right|^{q}
\end{array}\right)\right]^{\frac{1}{q}}\right] \\
\left.+C_{6}^{1-\frac{1}{q}}(\alpha)\left[\left(\begin{array}{c}
C_{7}(\alpha)\left|f^{\prime}(a)\right|^{q} \\
+C_{8}(\alpha)\left|f^{\prime}(b)\right|^{q}
\end{array}\right)\right]^{\frac{1}{q}}\right],
\end{array}\right.
\end{gathered}
$$

(3) If we take $\alpha=1$ and $g(x)=1$ we have the following Hermite-Hadamard type inequality for harmonically convex functions which is related to the left-hand side of (4):

$$
\begin{aligned}
& \left|f\left(\frac{2 a b}{a+b}\right)-\frac{a b}{b-a} \int_{a}^{b} \frac{f(x)}{x^{2}} d x\right| \\
& \quad \leq a b(b-a)\left[\begin{array}{c}
C_{3}^{1-\frac{1}{q}}(1)\left[\left(\begin{array}{c}
C_{4}(1)\left|f^{\prime}(a)\right|^{q} \\
+C_{5}(1)\left|f^{\prime}(b)\right|^{q}
\end{array}\right)\right]^{\frac{1}{q}} \\
+C_{6}^{1-\frac{1}{q}}(1)\left[\left(\begin{array}{c}
C_{7}(1)\left|f^{\prime}(a)\right|^{q} \\
+C_{8}(1)\left|f^{\prime}(b)\right|^{q}
\end{array}\right)\right]^{\frac{1}{q}}
\end{array}\right] .
\end{aligned}
$$

We can state another inequality for $q>1$ as follows:

Theorem 8 Let $f: I \subset(0, \infty) \rightarrow \mathbb{R}$ be a differentiable function on $I^{\circ}$, the interior of $I$, such that $f^{\prime} \in L[a, b]$, where $a, b \in I$. If $\left|f^{\prime}\right|^{q}, q>1$, is harmonically convex on $[a, b]$, $g:[a, b] \rightarrow \mathbb{R}$ is continuous and harmonically symmetric with respect to $\frac{2 a b}{a+b}$, then the following inequality for fractional integrals holds:

$$
\begin{aligned}
& \mid \begin{array}{l}
\frac{f(a)+f(b)}{2}\left[J_{1 / b+}^{\alpha}(g \circ h)(1 / a)+J_{1 / a-}^{\alpha}(g \circ h)(1 / b)\right] \\
-\left[J_{1 / b+}^{\alpha}(f g \circ h)(1 / a)+J_{1 / a-}^{\alpha}(f g \circ h)(1 / b)\right]
\end{array} \\
& \leq \frac{\|g\|_{\infty} a b(b-a)}{\Gamma(\alpha+1)}\left(\frac{b-a}{a b}\right)^{\alpha} \\
& \quad \times\left[\begin{array}{c}
C_{9}^{\frac{1}{p}}(\alpha)\left[\frac{\left|f^{\prime}(a)\right|^{q}+3\left|f^{\prime}(b)\right|^{q}}{8}\right]^{\frac{1}{q}} \\
+C_{10}^{\frac{1}{p}}(\alpha)\left[\frac{3\left|f^{\prime}(a)\right|^{q}+\left|f^{\prime}(b)\right|^{q}}{8}\right]^{\frac{1}{q}}
\end{array}\right]
\end{aligned}
$$


where

$$
\begin{aligned}
C_{9}(\alpha) & =\frac{(a+b)^{-2 p}}{2^{\alpha p-2 p+1}(\alpha p+1)}{ }_{2} F_{1}\left(2 p, 1 ; \alpha p+2 ; \frac{b-a}{b+a}\right), \\
C_{10}(\alpha) & =\frac{b^{-2 p}}{2^{\alpha p+1}(\alpha p+1)}{ }_{2} F_{1}\left(2, \alpha p+1 ; \alpha p+2 ; \frac{1}{2}\left(1-\frac{a}{b}\right)\right),
\end{aligned}
$$

with $\alpha>1, h(x)=1 / x, x \in\left[\frac{1}{b}, \frac{1}{a}\right]$ and $1 / p+1 / q=1$.

Proof Using (12), Hölder's inequality and the harmonically convexity of $\left|f^{\prime}\right|^{q}$, it follows that

$$
\begin{aligned}
& \left|\begin{array}{c}
f\left(\frac{2 a b}{a+b}\right)\left[J_{\frac{a+b}{2 a b}+}^{\alpha}(g \circ h)(1 / a)+J_{\frac{a+b}{2 a b}-}^{\alpha}(g \circ h)(1 / b)\right] \\
-\left[J_{\frac{a+b}{2 a b}+}^{\alpha}(f g \circ h)(1 / a)+J_{\frac{a+b}{2 a b}-}^{\alpha}(f g \circ h)(1 / b)\right]
\end{array}\right| \\
& \leq \frac{\|g\|_{\infty} a b(b-a)}{\Gamma(\alpha+1)}\left(\frac{b-a}{a b}\right)^{\alpha}\left[\begin{array}{c}
\int_{0}^{\frac{1}{2}} \frac{u^{\alpha}}{(u b+(1-u) a)^{2}}\left|f^{\prime}\left(\frac{a b}{u b+(1-u) a}\right)\right| d u \\
+\int_{\frac{1}{2}}^{1} \frac{(1-u)^{\alpha}}{(u b+(1-u) a)^{2}}\left|f^{\prime}\left(\frac{a b}{u b+(1-u) a}\right)\right| d u
\end{array}\right] \\
& \leq \frac{\|g\|_{\infty} a b(b-a)}{\Gamma(\alpha+1)}\left(\frac{b-a}{a b}\right)^{\alpha}\left[\begin{array}{c}
\left(\int_{0}^{\frac{1}{2}} \frac{u^{\alpha p}}{(u b+(1-u) a)^{2 p}} d u\right)^{\frac{1}{p}} \\
\times\left(\int_{0}^{\frac{1}{2}}\left|f^{\prime}\left(\frac{a b}{u b+(1-u) a}\right)\right|^{q} d u\right)^{\frac{1}{q}}
\end{array}\right. \\
& +\left(\int_{\frac{1}{2}}^{1} \frac{(1-u)^{\alpha p}}{(u b+(1-u) a)^{2 p}} d u\right)^{\frac{1}{p}} \\
& \times\left(\int_{\frac{1}{2}}^{1}\left|f^{\prime}\left(\frac{a b}{u b+(1-u) a}\right)\right|^{q} d u\right)^{\frac{1}{q}} \\
& \leq \frac{\|g\|_{\infty} a b(b-a)}{\Gamma(\alpha+1)}\left(\frac{b-a}{a b}\right)^{\alpha}\left[\begin{array}{c}
\left(\int_{0}^{\frac{1}{2}} \frac{u^{\alpha p}}{(u b+(1-u) a)^{2 p}} d u\right)^{\frac{1}{p}} \\
\times\left(\int_{0}^{\frac{1}{2}} u\left|f^{\prime}(a)\right|^{q}+(1-u)\left|f^{\prime}(b)\right|^{q} d u\right)^{\frac{1}{q}}
\end{array}\right. \\
& +\left(\int_{\frac{1}{2}}^{1} \frac{(1-u)^{\alpha p}}{(u b+(1-u) a)^{2 p}} d u\right)^{\frac{1}{p}} \\
& \times\left(\int_{\frac{1}{2}}^{1} u\left|f^{\prime}(a)\right|^{q}+(1-u)\left|f^{\prime}(b)\right|^{q} d u\right)^{\frac{1}{q}} \\
& =\frac{\|g\|_{\infty} a b(b-a)}{\Gamma(\alpha+1)}\left(\frac{b-a}{a b}\right)^{\alpha} \\
& \times\left[\left(\int_{0}^{\frac{1}{2}} \frac{u^{\alpha p}}{(u b+(1-u) a)^{2 p}} d u\right)^{\frac{1}{p}}\left[\frac{\left|f^{\prime}(a)\right|^{q}+3\left|f^{\prime}(b)\right|^{q}}{8}\right]^{\frac{1}{q}}\right. \\
& \left.+\left(\int_{\frac{1}{2}}^{1} \frac{(1-u)^{\alpha p}}{(u b+(1-u) a)^{2 p}} d u\right)^{\frac{1}{p}}\left[\frac{3\left|f^{\prime}(a)\right|^{q}+\left|f^{\prime}(b)\right|^{q}}{8}\right]^{\frac{1}{q}}\right] .
\end{aligned}
$$


For the appearing integrals, we have

$$
\begin{aligned}
\int_{0}^{\frac{1}{2}} \frac{u^{\alpha p}}{(u b+(1-u) a)^{2 p}} d u & =\frac{1}{2^{\alpha p+1}} \int_{0}^{1} \frac{u^{\alpha p}}{\left(\frac{u}{2} b+\left(1-\frac{u}{2}\right) a\right)^{2 p}} d u \\
& =\frac{1}{2^{\alpha p+1}} \int_{0}^{1}(1-v)^{\alpha p}\left(\frac{a+b}{2}\right)^{-2 p}\left[1-v\left(\frac{b-a}{b+a}\right)\right]^{-2 p} d v \\
& =\frac{(a+b)^{-2 p}}{2^{\alpha p-2 p+1}(\alpha p+1)}{ }_{2} F_{1}\left(2 p, 1 ; \alpha p+2 ; \frac{b-a}{b+a}\right) \\
& =C_{9}(\alpha) .
\end{aligned}
$$

Similarly, we have

$$
\begin{aligned}
\int_{\frac{1}{2}}^{1} \frac{(1-u)^{\alpha p}}{(u b+(1-u) a)^{2 p}} d u & =\int_{0}^{\frac{1}{2}} \frac{u^{\alpha p}}{(u a+(1-u) b)^{2 p}} d u \\
& =\frac{1}{2^{\alpha p+1}} \int_{0}^{1} \frac{u^{\alpha p}}{\left(\frac{u}{2} a+\left(1-\frac{u}{2}\right) b\right)^{2 p}} d u \\
& =\frac{1}{2^{\alpha p+1}} \int_{0}^{1} u^{\alpha} b^{-2 p}\left(1-\frac{u}{2}\left(1-\frac{a}{b}\right)\right)^{-2 p} d u \\
& =\frac{b^{-2 p}}{2^{\alpha p+1}(\alpha p+1)}{ }_{2} F_{1}\left(2, \alpha p+1 ; \alpha p+2 ; \frac{1}{2}\left(1-\frac{a}{b}\right)\right) \\
& =C_{10}(\alpha)
\end{aligned}
$$

If we use (27) and (28) in (26), we have (25). This completes the proof.

\section{Corollary 3 In Theorem 8:}

(1) If we take $\alpha=1$ we have the following Hermite-Hadamard-Fejer inequality for harmonically convex functions which is related to the left-hand side of (5):

$$
\begin{aligned}
& \left|f\left(\frac{2 a b}{a+b}\right) \int_{a}^{b} \frac{g(x)}{x^{2}} d x-\int_{a}^{b} \frac{f(x) g(x)}{x^{2}} d x\right| \\
& \quad \leq\|g\|_{\infty}(b-a)^{2}\left[\begin{array}{c}
C_{9}^{\frac{1}{p}}(1)\left[\frac{\left|f^{\prime}(a)\right|^{q}+3\left|f^{\prime}(b)\right|^{q}}{8}\right]^{\frac{1}{q}} \\
+C_{10}^{\frac{1}{p}}(1)\left[\frac{3\left|f^{\prime}(a)\right|^{q}+\left|f^{\prime}(b)\right|^{q}}{8}\right]^{\frac{1}{q}}
\end{array}\right],
\end{aligned}
$$

(2) If we take $g(x)=1$ we have following Hermite-Hadamard type inequality for harmonically convex functions in fractional integral forms which is related to the left-hand side of $(6)$ :

$$
\begin{aligned}
& \left|f\left(\frac{2 a b}{a+b}\right)-\frac{\Gamma(\alpha+1)}{2^{1-\alpha}}\left(\frac{a b}{b-a}\right)^{\alpha}\left\{\begin{array}{c}
J_{\frac{a+b}{2 a b}}^{\alpha}+(f \circ h)(1 / a) \\
+J_{\frac{a+b}{2 a b}-}^{\alpha}(f \circ h)(1 / b)
\end{array}\right\}\right| \\
& \quad \leq \frac{a b(b-a)}{2^{1-\alpha}}\left[\begin{array}{c}
C_{9}^{\frac{1}{p}}(\alpha)\left[\frac{\left|f^{\prime}(a)\right|^{q}+3\left|f^{\prime}(b)\right|^{q}}{8}\right]^{\frac{1}{q}} \\
+C_{10}^{\frac{1}{p}}(\alpha)\left[\frac{3\left|f^{\prime}(a)\right|^{q}+\left|f^{\prime}(b)\right|^{q}}{8}\right]^{\frac{1}{q}}
\end{array}\right],
\end{aligned}
$$


(3) If we take $\alpha=1$ and $g(x)=1$ we have the following Hermite-Hadamard type inequality for harmonically convex functions which is related to the left-hand side of (4):

$$
\begin{aligned}
& \left|f\left(\frac{2 a b}{a+b}\right)-\frac{a b}{b-a} \int_{a}^{b} \frac{f(x)}{x^{2}} d x\right| \\
& \quad \leq a b(b-a)\left[\begin{array}{c}
C_{9}^{\frac{1}{p}}(1)\left[\frac{\left|f^{\prime}(a)\right|^{q}+3\left|f^{\prime}(b)\right|^{q}}{8}\right]^{\frac{1}{q}} \\
+C_{10}^{\frac{1}{p}}(1)\left[\frac{3\left|f^{\prime}(a)\right|^{q}+\left|f^{\prime}(b)\right|^{q}}{8}\right]^{\frac{1}{q}}
\end{array}\right] .
\end{aligned}
$$

\section{Conclusion}

In this paper, new Hermite-Hadamard type inequalities for harmonically convex functions in fractional integral forms are given and Hermite-Hadamard-Fejer inequalities for harmonically convex functions in fractional integral forms are built. Also, an integral identity and some Hermite-Hadamard-Fejer type integral inequalities for harmonically convex functions in fractional integral forms are obtained.

\section{Authors' contributions}

MK, II, NY, UG contributed equally to the writing of this paper. All authors read and approved the final manuscript.

\section{Author details}

${ }^{1}$ Department of Mathematics, Faculty of Sciences, Karadeniz Technical University, Trabzon, Turkey. ${ }^{2}$ Department of Mathematics, Faculty of Sciences and Arts, Giresun University, Giresun, Turkey.

\section{Acknowledgements}

The authors are very grateful to the referees for helpful comments and valuable suggestions. Also, Kunt and Isscan are very grateful to their PhD supervisor Prof. Dr. Abdullah Çavuş.

\section{Competing interests}

The authors declare that they have no competing interests.

Received: 9 February 2016 Accepted: 22 April 2016

Published online: 17 May 2016

\section{References}

Bombardelli M, Varošanec S (1869) Properties of h-convex functions related to the Hermite-Hadamard-Fejér inequalities. Comput Math Appl 58(2009):1877

Chen F, Wu S (2014) Fejer and Hermite-Hadamard type inqequalities for harmonically convex functions. J Appl Math 2014, article id: 386806

Dahmani Z (2010) On Minkowski and Hermite-Hadamard integral inequalities via fractional integration. Ann Funct Anal 1(1):51-58

Fejér L (1906) Uber die Fourierreihen, II, Math. Naturwise. AnzUngar. Akad., Wiss, 24:369-390 (in Hungarian)

Hadamard J (1893) Étude sur les propriétés des fonctions entières et en particulier d'une fonction considérée par Riemann. J Math Pures Appl 58:171-215

Hermite Ch (1883) Sur deux limites d'une intégrale définie. Mathesis 3:82-83

Işscan I (2013a) New estimates on generalization of some integral inequalities for s-convex functions and their applications. Int J Pure Appl Math 86(4):727-746

İscan I (2013b) Generalization of different type integral inequalities for s-convex functions via fractional integrals. Applicable Analysis. doi:10.1080/00036811.2013.851785

İşcan I (2014a) On generalization of different type integral inequalities for s-convex functions via fractional integrals. Math Sci Appl E-Notes 2(1):55-67

Işcan I (2014b) Hermite-Hadamard type inequalities for harmonically convex functions. Hacet J Math Stat 43(6):935-942

İscan I (2014c) Some new general integral inequalities for h-convex and h-concave functions. Adv Pure Appl Math 5(1):21-29. doi:10.1515/apam-2013-0029

Isşcan I, Wu S (2014) Hermite-Hadamard type inequalities for harmonically convex functions via fractional integrals. Appl Math Comput 238:237-244

Kilbas AA, Srivastava HM, Trujillo JJ (2006) Theory and applications of fractional differential equations. Elsevier, Amsterdam

Latif MA, Dragomir SS, Momoniat E (2015) Some Fejer type inequalities for harmonically-convex functions with applications to special means. http://rgmia.org/papers/v18/v18a24

Mihai MV, Ion DA (2014) Generalization of some inequalities via Riemann-Liouville fractional calculus. Tamkang J Math 45(2):207-215 
Minculete N, Mitroi F-C (2012) Fejér type inequalities. Aust J Math Anal Appl 9(1):1-8 (Article 12)

Prudnikov AP, Brychkov YA, Marichev OJ (1981) Integral and series, elementary Functions, vol 1. Nauka, Moscow

Sarıkaya MZ (2012) On new Hermite Hadamard Fejér type integral inequalities. Stud Univ Babeş-Bolyai Math 57(3):377-386

Sarıkaya MZ, Set E, Yaldız H, Başak N (2013) Hermite-Hadamard's inequalities for fractional integrals and related fractional inequalities. Math Comput Model 57(9):2403-2407

Tseng K-L, Yang G-S, Hsu K-C (2011) Some inequalities for differentiable mappings and applications to Fejér inequality and weighted trapezoidal formula. Taiwan J Math 15(4):1737-1747

Wang J, Li X, Fečkan M, Zhou Y (2012) Hermite-Hadamard-type inequalities for Riemann-Liouville fractional integrals via two kinds of convexity. Appl Anal 92(11):2241-2253. doi:10.1080/00036811.2012.727986

Wang J, Zhu C (2013) New generalized Hermite-Hadamard type inequalities and applications to special means. J Inequal Appl 2013(325):1-15

\section{Submit your manuscript to a SpringerOpen ${ }^{\circ}$} journal and benefit from:

- Convenient online submission

\section{- Rigorous peer review}

- Immediate publication on acceptance

- Open access: articles freely available online

- High visibility within the field

- Retaining the copyright to your article

Submit your next manuscript at $\gg$ springeropen.com 